\title{
Stability of mitotic spindle using computational mechanics
}

\author{
Andrii lakovliev \\ School of Electronics and Computer \\ Science. University of Southampton. \\ University Road, Southampton, UK \\ $+44(0) 7479045846$ \\ A.lakovliev@soton.ac.uk
}

\author{
Srinandan Dasmahapatra \\ School of Electronics and Computer \\ Science. University of Southampton. \\ University Road, Southampton, UK \\ $+44(0) 2380594503$ \\ sd@ecs.soton.ac.uk
}

\author{
Atul Bhaskar \\ Faculty of Engineering and the \\ Environment. University of \\ Southampton. \\ University Road, Southampton, UK \\ $+44(0) 2380593825$
}

A.Bhaskar@soton.ac.uk

\begin{abstract}
Fidelity of cell division depends on the ability of an internal cell structure called the mitotic spindle, to maintain the structural integrity of the cellular architecture despite being subject to high compressive loading. We propose a generic software tool called Spindle FEA that employs continuum mechanics and finite elements analysis (FEA) code Abaqus CAE to study the stability of mitotic spindles in various phases of mitosis. The proposed application has a modular structure which allows easy modification of any part of the analysis which is of particular importance considering that new knowledge of spindles is constantly emerging. Thanks to the highly optimised finite element solver used in Abaqus CAE, Spindle FEA is highly suitable for large multiparametric studies which in turn may significantly benefit the planning of new experiments or identifying new key properties of the spindle. We also discuss the main physiological properties of spindles and show how they are modelled with the proposed technique as well as discuss all the essential analysis steps. We use Spindle FEA to study the buckling of a mitotic spindle in anaphase $\mathrm{B}$ to show how the additional stiffness of the lateral support of the spindle affects the left-right symmetry of cell division as well as to demonstrate the capacities of the proposed technique.
\end{abstract}

\section{CCS Concepts}

Applied computing $\rightarrow$ Computer-aided design; Molecular structural biology;

\section{Keywords}

Cell division; mitotic spindle stability; anaphase B.

\section{INTRODUCTION}

The mitotic spindle is a cytoskeletal structure that plays a crucial role in cell division (mitosis) by generating forces needed to separate the chromosomes while preserving cell shape under external loads and ensuring correct positioning of the nucleus. The structural performance of spindles is partially facilitated by their highly complex architecture that combines various proteins arranged in the form of microtubules (MTs), cross-linkers (connectors), protein motors, centrosomes (poles), chromosomes and others. Investigation of the arrangement, properties and interaction of these structural units is essential to understand the high load bearing capacity of the spindle and the consequent robustness of the biological functions that rely on its mechanical stability.

Permission to make digital or hard copies of all or part of this work for personal or classroom use is granted without fee provided that copies are not made or distributed for profit or commercial advantage and that copies bear this notice and the full citation on the first page. To copy otherwise, or republish, to post on servers or to redistribute to lists, requires prior specific permission and/or a fee.
Many experimental [1-4] and theoretical studies [5-8] at various levels of spindle organisation and length scales address the questions of force generation, structural performance and stability of spindles. They include the near atomic level studies of MT connectors and motors [9], investigation of the structural properties of individual MTs [10-13] as well as analysis of MT organisation [1], coupling and positioning $[6,14,15]$. Studies of the spindle as a whole address spindle structure and orientation within the cell $[2,16,17]$ and estimation of forces arising within the spindle as well as mechanisms of spindle formation [18].

The studies focused on creating some unified models of the spindle which incorporate the properties of each structural unit into a complete hierarchical model of the whole spindle are rather incomplete. One such generic model of the inter-polar bundle of MTs in the fission yeast cells is proposed by Ward et al. [2] and is based on the Langevin dynamics framework [19]. Other models aim at producing closed form analytical solutions or scaling laws to explain force generation and structural stability of the spindle under compression $[7,8,20]$. These studies are of particular interest to us as they lay the foundation for the understanding of the structural behaviour of the spindle as a whole and, to some extent, incorporate all its hierarchical complexity. Furthermore, they clearly make the case for the importance of structural models of the spindle as a whole in scaffolding biological mechanism. In addition to this, the global models of the spindle are mostly tailored to some specific cell types and/or particular phases of mitosis. Motivated by this, we propose an alternative approach to structural modelling of the spindle as a whole. Here, we lay the foundation for a generic computational framework based on continuum mechanics methods and the theory of elastic stability which deploys Abaqus CAE finite element analysis (FEA) code to studying structural response of spindles. The proposed framework is embedded in the developed application Spindle FEA [21] which is linked to Abaqus CAE code via an API and receives a list of spindle parameters from a user, generates a Finite Element (FE) model of a spindle and submits it to FEA solver for analysis. In this paper, we discuss a high-level architecture of the developed application, formulate essential assumptions and limitations, and employ Spindle FEA to study the elastic stability of the fission yeast spindle in late anaphase B. Then we compare our results with those by Ward et al. [27] and discuss the applicability of the proposed framework. Our long-term goal is to create an open source modular platform that will be used and extended by the community of researchers and incorporate continuously emerging new knowledge about spindles.

We show that at the current development stage, Spindle FEA application based on the continuum mechanics framework is a highly customisable and versatile tool that can be tailored to model many structural aspects of mitotic spindles in various cell types. 


\section{ARCHITECTURE OF THE APPLICATION}

The basic requirements that we pose to our application are the ability to model the hierarchical complexity of a mitotic spindle, applicability to various cell types and phases of mitosis, extensibility, and scalability \& simplicity of use. We aim to develop a platform that can be used by researchers in the field and be extended by them to account for new experimental and theoretical findings as well as to give researchers a numerical tool that they can use to test various modelling assumptions.

The application is based on continuum mechanics discretised using a finite element (FE) method in quasi-static approximation. Continuum mechanics at a length scale of dozens of nanometres (length scale of an MT cross-section) has been successfully used before $[6,15,22]$ demonstrating its applicability at such scales. In classical mechanics, finite element analysis (FEA) has long become a standard tool for computer-aided design and modelling of structures, especially when the geometry is complex and/or the boundary conditions are difficult for analytical treatment. The benefits of FEA are the high versatility of modelling, the applicability of the methods to virtually any structural mechanics problem and simplicity of its use. Therefore, at the current stage, in Spindle FEA we employ a highly optimised, efficient and reliable commercial FE solver such as Abaqus CAE. The reason for using this closed source solver is that it is one of the industry standards in FE analysis and also provides a convenient Python 2.7 API to control the solver. In the further releases we will aim to adjust the application to work with other open-source FE solvers but after their ability to deal with spindle stability problems is thoroughly tested.

Spindle geometry generation, material properties definition, FE meshing and model formulation is triggered in Spindle FEA and executed in Abaqus CAE. Such formulated problem is then wrapped in the form of a specific input file which is passed to the FE solver for analysis. The results of the calculations can be then extracted and visualised either in the Abaqus graphical user interface or using python data tools. In the basic use scenario, the user provides the essential model parameters in a single file "job.py" located at the root of application and executes a single command line command. The model generation and analysis are then done automatically. In such a way, altering the geometry of the spindle, and/or material properties of its parts and running multi-parameter studies is as easy as changing the appropriate parameters in "job.py" file. In the case of a more advanced use, new material models, geometric and/or material nonlinearities and new types of analysis can also be incorporated into the framework by modifying the existing modules or adding new ones.

The detailed interactive user manual for Spindle FEA explaining the steps needed to run the analysis as well as to introduce basic configurational changes can be found within the Spindle FEA directory [21] by following the simple guide in "README.txt" file. Alternatively, the application directory can be downloaded or cloned from https://git.soton.ac.uk/ai1v14/SpindleFEA.git and the interactive documentation can also be accessed and cloned from https:/git.soton.ac.uk/ai1v14/SpindleFEA-docs.git. A website with the interactive documentation as well as modelling examples is spindlefea.soton.ac.uk. The link to it will be added to a "README" file in https://git.soton.ac.uk/ai1v14/SpindleFEA.git

\subsection{Main assumptions}

Some of the further discussed assumptions and limitations are inherited from the continuum mechanics and FEA, others come from the physics and biology of a mitotic spindle. Many of these assumptions are prone to changes as the understanding of the problem evolves and thus new material, geometry models, physical and physiological properties may be introduced by the users in the future.

We assume that all the structural parts of the spindle are solid and continuous bodies and that the atomic and molecular thermal fluctuations are neglected. As we are currently focused on the various scenarios in which a spindle becomes structurally unstable and buckles, only linear buckling is considered. At this stage we assume all spindle parts to be linearly elastic implying a linear stress-strain relationship defined by Hooke's law also assuming all deflections and rotations to be small.

The mechanical model of MTs and connectors is an isotropic Bernoulli-Euler beam [23]. Although, several recent studies indicate that the orthotropic beam model for the MTs may be more realistic $[10,13]$, we use the isotropic model due to its simplicity. Going beyond the assumptions of the models used in this paper orthotropic models, geometric and material nonlinearities, as well as post-buckling analysis, may be also incorporated relatively easily as such problems are typically studied by FEA.

The implementation of the quasi-static assumption allows us to perform linear buckling analysis of the spindle without dealing with the complicated step-dependent nonlinear buckling analysis or dynamical analysis as done by Ward et al. [2]. The validity of the quasi-static assumption requires the slow evolution of the spindle in time. Thus, loads and deformations develop slowly and the forces are in equilibrium at each time snapshot of the mitosis which typically spans over time interval of dozens of minutes [2] and the each of the mitotic phases changes slowly over a duration of several minutes. Thus, we are able to neglect the time dependence of the problem and apply the linear theory of elastic stability.

Finally, in our use of finite element analysis we divide the spindle into a number of small sub-structures (finite elements) connected at nodes whose displacements are described by assumed shape functions used for the interpolation of the displacement field within each element; the shape being controlled by a set of relatively small number of generalised coordinates. These displacements are approximated by polynomial shape functions that satisfy variational constraints on the energy of the system. The matching of the displacements, strains, stresses, etc., between elements, is ensured by continuity conditions. In the case of linear buckling analysis, the FEA returns critical buckling loads of the structure and the associated instability shapes or modes.

\subsection{Mitotic Spindle structure}

Consider the scheme of the mitotic spindle structure shown in Figure 1 (A). The red arrows indicate the direction of the loads generated by protein motors pushing against the antiparallel assembly of inter-polar MTs (ipMTs) indicated by purple and green lines. The purple lines indicate MTs growing from the left pole while the green ones indicate MTs growing from the right pole. A detailed view of the mid-zone and near-pole parts of the inter-polar assembly is shown in Figure 1 (B) and Figure 1 (C). The inter-polar MTs translate the forces generated by motor proteins to the centrosomes or poles indicated by two big blue circles in Figure 1 (A), causing the two poles to separate. The poles are supported inside the cell by a number of radially oriented astral MTs (aMTs) indicated by blue thick lines in Figure 1 (A). Astral MTs connect centrosomes to the membrane of the cell and experience forces transmitted by the poles as well as reaction forces from membrane resistance to expansion as indicated by blue arrows 
in Figure 1 (A). Furthermore, centrosomes are also connected to both halves of the chromosomes (orange bodies in Figure 1 (A)) by means of chromosomal MTs (chMTs) indicated by thin cyan lines. Thus, under the influence of protein motors the centrosomes move apart, pushing the membrane via the attached aMTs and pulling the chromosomes apart due to chMTs. The direction of the chromosome splitting loads is indicated with orange arrows. In the case of late anaphase B, chromosomes have been already split apart so we do not account for chromosome splitting forces.

Note that the actual spindle of a living cell contains hundreds of different MTs and other bodies. The scheme in Figure 1 (A), Figure 1 (B) and Figure 1 (C) is simplified to show only the highlevel architecture of the spindle and thus emphasise the key structural elements modelled here. A photo of the actual spindle reconstructed from the electron microscopy was adapted from [17] and is shown in Figure 1 (D) to demonstrate how complex the actual spindle is. The white lines are the individual MTs and the black circles indicate the poles.

Following the scheme in Figure 1 (A) Spindle FEA generates a structure of a mitotic spindle depicted in Figure 2 (A). Here the centrosomes are indicated with the large red circles, the astral MTs and inter-polar MTs are represented by light-blue lines and the midzone where the MTs are connected by cross-linkers is shown in green. The detailed view of the mid-zone is shown in Figure 2 (B) depicting inter-polar MTs as well as connectors and motors. The zoomed view of a connector and an MT are shown in Figure 2 (C) and Figure 2 (D). The load $\mathrm{P}$ arising from motor push is shown with black arrows in Figure $2(\mathrm{C})$. The centrosome with the radially oriented astral MTs is depicted in Figure 2 (E).

In the model presented here, we reduce the complex structural behaviour of the spindle to a problem of stability of equilibrium of the elastic system consisting of elastically coupled flexible bodies. The initial state of the system is taken to be in equilibrium. When loading is quasi-statically increased it reaches the critical point after which a pitch-fork bifurcation occurs, leading to symmetry breaking via buckling mode. We are interested in the onset of buckling and in the dependence of buckling loads and corresponding deformed configurations of the spindle on spindle parameters. Hence, in order to be able to study this problem, we first need to discuss the structure and mechanical behaviour of each individual part of a typical spindle.

\subsection{Structure and properties of a microtubule}

Microtubules are hollow filaments with cylindrical cross-section $[5,10,13]$. The outer diameter of a typical MT is $D \approx 25 \mathrm{~nm}$ while its inner diameter $d \approx 15 \mathrm{~nm}$ as indicated in Figure $1(\mathrm{~F})$. The length of MTs varies depending on the cell type and is between $1-$ $10 \mu \mathrm{m}$ to $50-100 \mu \mathrm{m}$. Stiffness of MTs is derived from the characteristic length scale called persistence length which is measured in experiments [10], [23] and represents a ratio of MT bending stiffness $\kappa$ to thermal noise $k_{B} T$ written as $l_{p}=\kappa / k_{B} T$; here $\kappa$ is the bending moment per unit curvature response. The simplest mechanical model of an MT that has been often used in literature $[6,15,22]$ is based on isotropic Bernoulli-Euler beam. However, the values of persistence length measured in experiments $[24,25]$ indicate that an orthotropic beam model with shear effects would more accurately capture the MT mechanics when MT length $L<21 \mu \mathrm{m}[10]$. In the current study we restrain ourselves to the isotropic model with the orthotropic MT model to be incorporated in the near future.

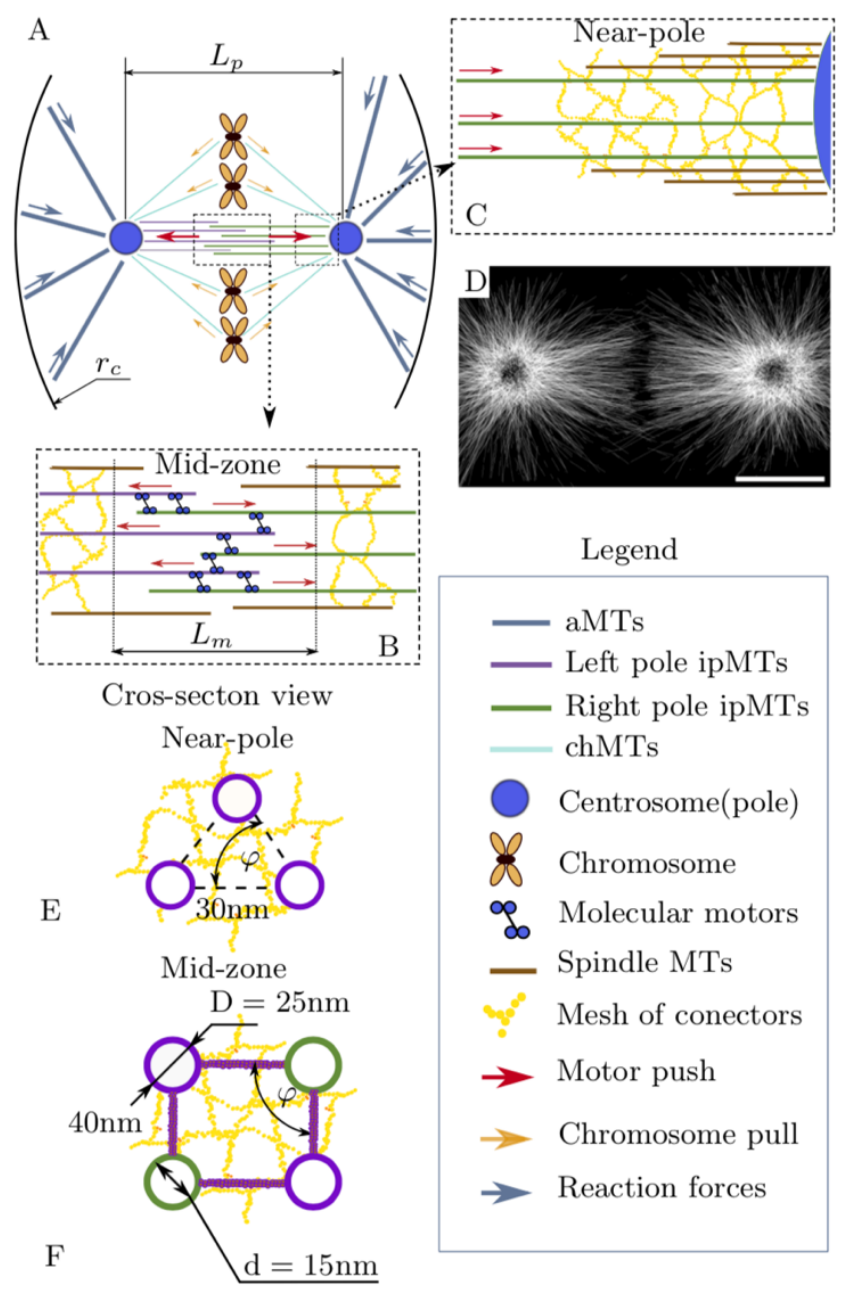

Figure 1. Mitotic spindle architecture at various hierarchical levels. Forces acting in spindle are shown in (A) along with the cell membrane, centrosomes, chromosomes, chromosome MTs (chMTs), astral MTs (aMTs) and inter-polar MTs (ipMTs). The magnified view of the spindle mid-zone with protein motors and mesh of MT connectors reported in [17] are shown in (B). The inter-polar MTs along with the connector mesh near poles are shown in (C). Red arrows indicate the direction of protein motors push. The view of the spindle constructed from electron microscopy data is adapted from [15] and shown in (D) The cross-section of inter-polar MT bundles as reported in [27] near pole is shown in (E) while the cross-section of the inter-polar MT bundle in mid-zone as reported in [27] is shown in (F). The crosssection architecture was enriched by considering that interpolar bundle is embedded in a mesh of connectors as reported in [17]. The motor proteins are omitted in (E) and (F) to simplify the scheme.

Typically, MTs that form a spindle are prone to large levels of compression due to forces generated by motor proteins that may destabilise them and cause buckling. In contrast to the typical beam buckling, MTs retain a considerable level of initial load bearing capacity even when buckled $[6,15]$. This may be explained by the effect of a short wave-length buckling occurring thanks to the extra support that is provided by the cross-linkers. Furthermore, it has been shown that MTs in a living cell are also embedded in the mesh of connectors attaching them to the rest of the cytoskeleton and 
providing considerable lateral support [3] (see Figure 1 (B) and Figure $1(\mathrm{C})$ ) which also leads to the short-wave buckling mode.

We model MTs as long slender cylindrical elastic beams with circular cross-section as shown in Figure 1 (E) and Figure 1 (D). We employ the isotropic Bernoulli-Euler beam model. Every MT is defined by length $L$ and cross-sectional stiffness $E I$ as shown in Figure 2 (D). The MTs are embedded within the cytoskeleton by means of a dense network of protein connectors that we model using elastic springs $k$ uniformly distributed along the MT contour as shown in Figure 2 (C) and Figure 2 (D).

Two types of MTs participate in a spindle, namely, inter- polar MTs in a pole-to-pole bundle which has length $\approx 10 \mu \mathrm{m}$ in late anaphase B and astral MTs that extend from poles to the membrane and are $\approx 2 \mu \mathrm{m}$ long. The locations of astral MTs are randomly generated on the centrosome sphere as shown in Figure 2 (A) and Figure 2 (E). The inter-polar bundle has a well-defined morphology presented in Figure 2 (B). The antiparallel alignment of MTs in a bundle implies that MTs do not span the entire inter-polar distance but grow towards each other from opposite poles and meet in the mid-zone where they are linked by cross-linkers and protein motors as shown in Figure 2 (A) and Figure 2 (B) as well as in Figure 1 (B) and Figure $1(\mathrm{C})$. The cross-sectional assembly of ipMTs is defined by the inter MT distance $\delta$ and MT angle $\varphi$ as shown in Figure 1 (E) and Figure $1(\mathrm{~F})$. For our modelling, we use values of $\delta$ and $\varphi$ generated by the Gaussian distribution with mean and standard deviation taken from the spindle measurements performed by Ward et al. [2]. These parameters, as well as other model parameters, are given in Table A of Appendix A. The length of the inter-polar MTs is also picked from a Gaussian distribution defined by mean and standard deviation given in in Table A of Appendix A. The inter-polar MTs grow from the centrosomes and thus are rigidly attached to them. In the model, it is represented by imposing continuity of displacements and rotations between the centrosomes and the ipMTs ends. The number of ipMTs in late anaphase B is between 5 and 6 [2].

\subsection{Cross-linkers and protein motors}

Consider a zone of overlap in the inter-polar bundle where MTs are strongly cross-linked by actin-binding and kinesin proteins $[3,26]$ as shown in Figure 1 (B). The protein motors push the antiparallel MTs into the opposite directions thus separating poles. Fidelity of this mechanism as well as structural integrity of the inter-polar bundle are crucial for mitosis and, thus, need to be accounted for by our modelling.

In the model both tubulin motors and other cross-linkers are regarded as thin $6-40 \mathrm{~nm}$ long struts randomly distributed along the mid-zone. Ward et al. [2] adopt from [26] the assumption that cross-linkers do not contribute to bending stiffness of the MT bundle. Yet, we assume that they have a finite but small thickness of $\approx 5 \mathrm{~nm}$. This allows us to have the cross-linker radius as another arbitrarily small parameter which we believe would represent the actual physics of the connector more accurately. It was shown [9], [3] that connectors are well coupled to the adjacent MTs which allows us to assume continuity of displacement and rotation between the adjacent nodes of the connector and the MT in the FE model. The estimated number of cross-linkers coupling inter-polar MTs varies in a wide range and they are randomly distributed along the MT length. The detailed scheme of the inter-polar bundle featuring dozens of connectors randomly distributed along five ipMTs is shown in Figure 2 (B). The detailed view of the connector linking two MTs is depicted in Figure 2 (C). The direction of force generated by the protein motor is shown with vectors $\boldsymbol{P}$ and the connector length $L_{c}$ as well as cross-sectional stiffness $E I_{c}$ are indicated in the scheme.

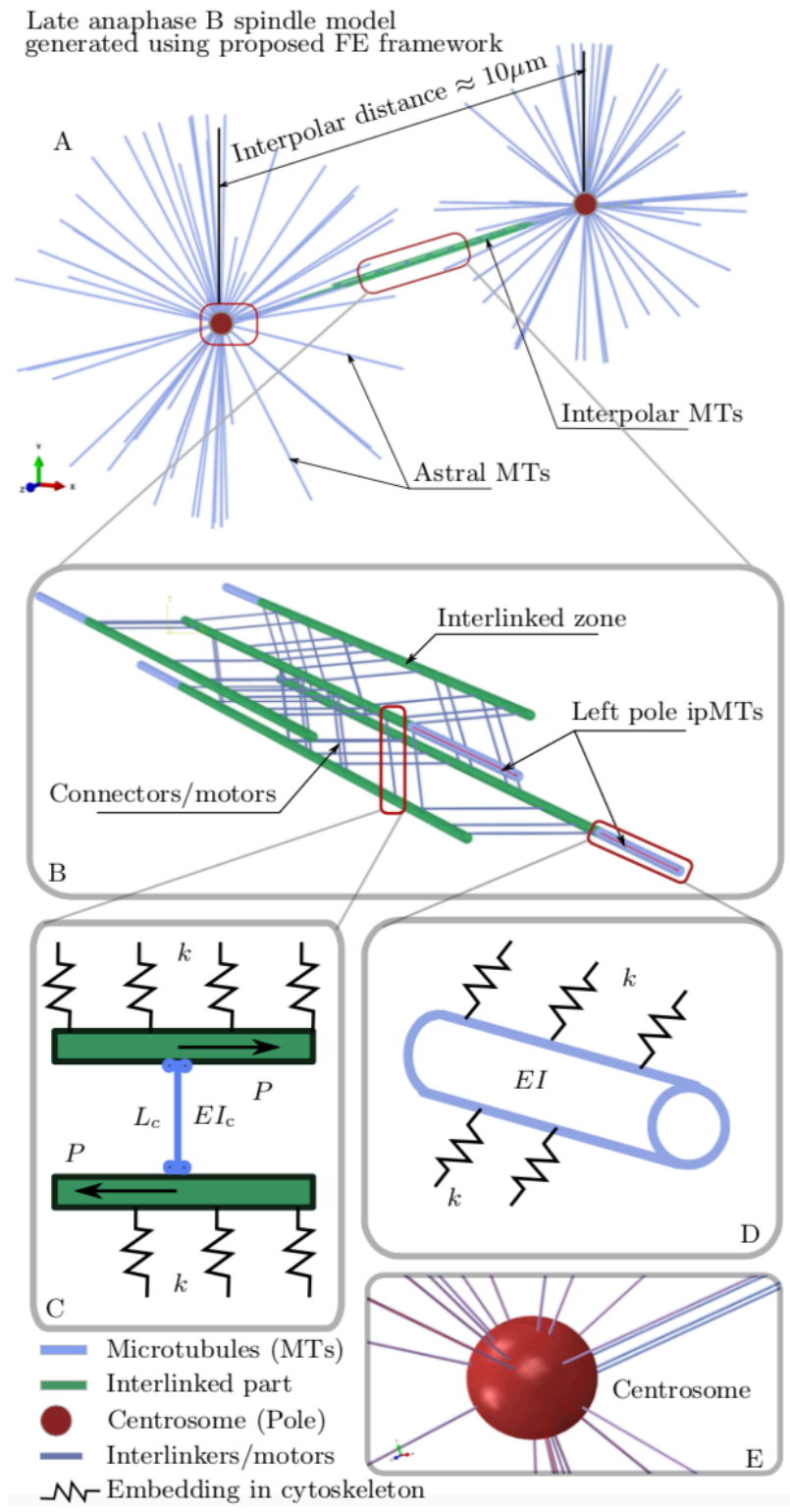

Figure 2. Late anaphase B mitotic spindle FE model. The generic architecture with centrosomes (red), inter-polar and astral MTs (blue) are shown in (A). The parts of the ipMTs interlinked by bridges and protein motors are green. The detailed view of the inter-polar zone is shown in (B). This blue links between MTs are MT connectors and motor proteins. The magnified view of a connector is shown in (C). Here, MTs are green and connector/motor is a blue link. When the motors walk in MTs they create two antiparallel loads $P$ shown with arrows. The connector length is $L_{c}$ and stiffness is $E I c$. The MTs have stiffness $E I$ and are also embedded in a highly chaotic mesh of cytoskeletal proteins. The effect of such embedding is modelled using distributed elastic springs $k$ which are shown in (C) and (D). The detailed view of the centrosome with the radially extending astral MTs is shown in (E).

Brangwynne et al. [15] have shown that individual microtubules bear up to 100 times larger compression loads in leaving cells. 
Also, in vivo buckling wavelengths of MTs appear much shorter than those predicted for isolated MTs [15,22]. It is argued that MTs in the cell are mechanically coupled not only to each other but also to the surrounding cytoskeleton by a mesh of cross-linkers which adds considerable stiffness to the MTs and contributes to shortwave buckling and to a dramatic increase in buckling loads compared to isolated MTs [3]. This network or mesh of proteins surrounding the MTs and linking them to the rest of the cytoskeleton has been observed using $3 \mathrm{D}$ reconstruction of electron microscopy data [3]. It was reported that this network has a chaotic morphology and can link 2, 3 or even 4 MTs at once which is shown in Figure 1 (B), Figure 1 (C), Figure 1 (E) and Figure 1 (F) with the help of yellow and orange dots.

We model the effect of the embedding of MTs into a cytoskeletal mesh by attaching an evenly distributed elastic springs $k$ (stiffness per unit length) to the contours of MTs while the second ends of the springs are attached to some fixed points in space mimicking high rigidity of the cytoskeleton of the cell. Therefore, the distributed spring contributes to the stiffness of each individual MT. The proposed distributed springs are sketched in Figure $2(\mathrm{C})$ and Figure 2 (D). We further omit displaying these springs to simplify the graphics.

\subsection{Spindle analysis algorithm}

The standard modelling case of a mitotic spindle employing Spindle FEA contains the following steps.

3. User specifies all the spindle analysis parameters in the "job.py" file located in the root of the application directory. The example of the user input data used in our modelling is shown in Table A of Appendix A and in the interactive user manual.

4. In "job.py" user may also specify the computation parameters such as number of CPUs requested, memory allocation and parallelisation algorithms.

5. The analysis may be run either from Abaqus graphical user interface or from command line. The "job.py" file is then submitted as an input file for the application.

6. The modelling starts by generating the geometry of individual parts of the spindle such as MTs, cross-linkers and centrosomes.

7. The material properties are then assigned to each part.

8. The parts are combined in the mitotic spindle assembly.

9. The connection properties between parts are specified.

10. The finite element mesh is generated on each part of the spindle.

11. The analysis input file is generated and submitted to the FE solver which formulates the generalised eigenvalue problem for the whole structure and works out the eigenvalues which represent the critical buckling loads and the eigenvectors that describe the buckling mode of the spindle.

12. The analysis is terminated and the files with results "job.odb" and "job.dat" are written and can be manipulated with either from Abaqus GUI or directly executing python code.

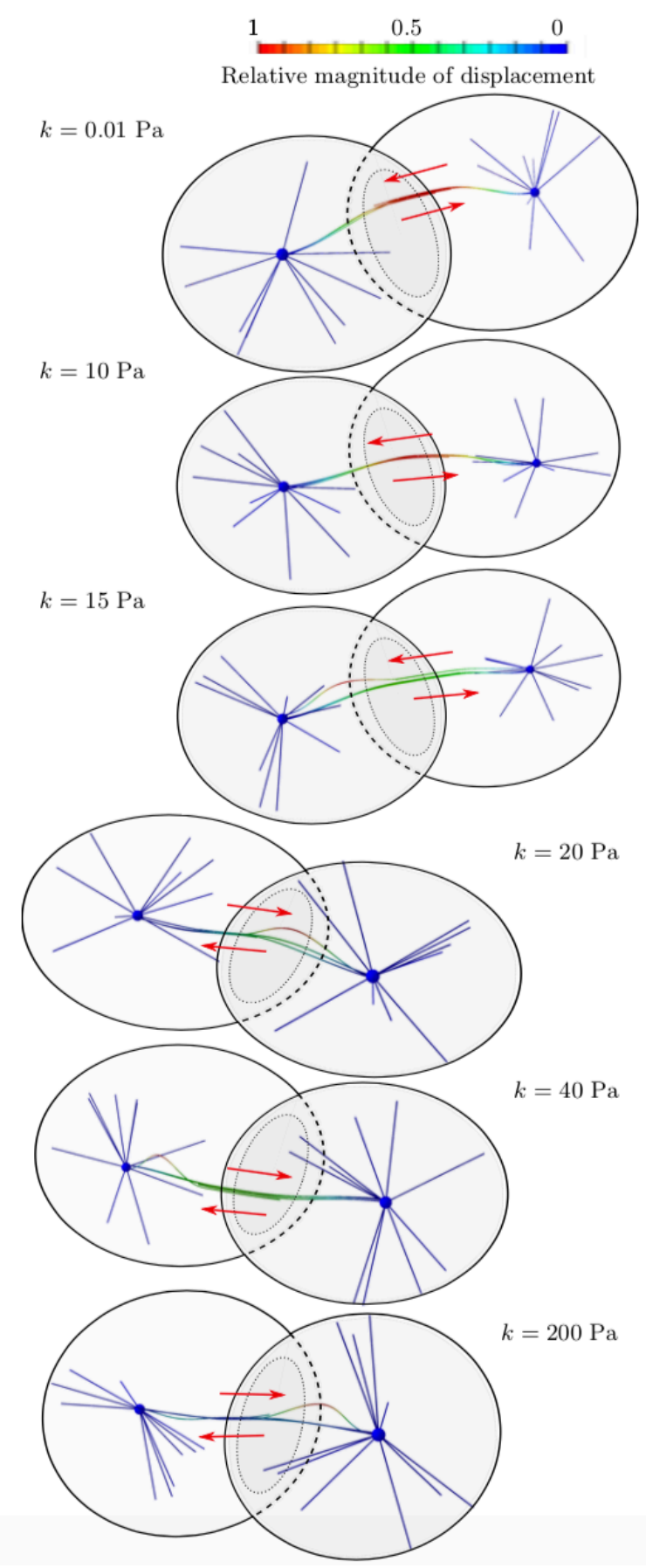

Figure 3. Buckling modes of late anaphase B mitotic spindle for various lateral support stiffness $k$. The colour code represents the non-dimensional relative displacement amplitude of the buckling mode with near-zero deflection being blue and the near-one deflection labelled with red colour.

\section{STABILITY OF A MITOTIC SPINDLE IN ANAPHASE B}

The stiffness added to the inter-polar MT bundle due to coupling with the rest of the cell cytoskeleton is challenging to measure in an experiment. Hence, we assume it as the governing parameter of our study and, thus, investigate how it affects the deformation profile of the late anaphase B spindle in buckling. We perform a 
parametric study of the dependence of the buckling and left-right symmetric properties of the spindle on the stiffness $k$ of the embedding of the inter-polar MT bundle in the surrounding cytoskeleton. This is also a test case for the utility of our tool Spindle FEA. In contrast to [2] we consider not only the inter-polar MT bundle but also astral MTs which are attached to the centrosomes and to the cell membrane. Six cases defined by $k$ being equal to $0.01 \mathrm{~Pa}, 10 \mathrm{~Pa}, 15 \mathrm{~Pa}, 20 \mathrm{~Pa}, 40 \mathrm{~Pa}$ and $200 \mathrm{~Pa}$ were considered.

The computed deformed configurations of the spindle are presented in Figure 3 for various values of lateral restoring force constant $k$. Each deformed configuration is characterised by the mode shape which is obtained from the eigenvector of the generalised eigenvalue problem constructed using the minimisation of the total potential energy according to the FE method. The eigenvector is determined up to an unknown scaling constant or amplitude of the buckling mode. Therefore, the analysis returns the shape of the deformation profile of the beam normalised to unity rather than the actual displacement in meters. The correspondent eigenvalue is, however, a physical critical buckling load in pN. It is essential to calculate the critical buckling loads of the spindle and the associated buckling modes as they give an estimate of the deformation pattern developing in a spindle should it be loaded to a particular critical load magnitude.

In Figure 3 a large relative displacement is indicated in red while the small relative displacement is shown in blue. The black circles represent the cell membrane which starts to split in the middle to form two new cells. The dotted ellipse shows the central plane of cell division with respect to which the division may be symmetric or asymmetric. The red arrows indicate the direction of the antiparallel push by protein motors.

From Figure 3, we observe that for $k=0.01 \mathrm{~Pa}$ the buckling mode of the inter-polar bundle resembles the Euler mode of the fixed-fixed beam-column [23] and also resembles the one reported in [2] for $k=0 \mathrm{~Pa}$. In this case, the lateral reinforcement of the bundle is neglectfully small and the bundle forms an arc symmetric with respect to the central plain.

When $k=10 \mathrm{~Pa}$, we see that the deformation profile starts to alter. The maximum displacement (shown in red) shifts slightly towards the left pole. As the support stiffness increases to $k=$ $15 \mathrm{~Pa}$ we observe that the symmetric arc is no longer present. The high curvature zone indicated in red is now fully shifted to the left pole indicating the possibility of a violation of the division symmetry with respect to the central plane. In this case, the motor push may be unevenly distributed between the left and the right halves of the spindle. Also note, the local buckling of one or two MTs prevails over the global buckling mode of the whole spindle. When $k=20 \mathrm{~Pa}$ and then $k=40 \mathrm{~Pa}$ this effect becomes even more prominent clearly demonstrating asymmetric buckling with respect to the central plane. Furthermore, the wavelength of the buckling mode decreases with $k$. In the case of $k=0.01 \mathrm{~Pa}$, the single arc extends over the whole bundle. However, for $k=20 \mathrm{~Pa}$ the arc spans roughly a half of the bundle. When $k=40 \mathrm{~Pa}$ the arc is even shorter while the deformation of the whole bundle is strongly reduced. When $k=200 \mathrm{~Pa}$ the two arcs buckling mode is observed and the global mode of the whole bundle is mostly absent. This leads to the conclusion that the lateral support transforms the global long-wavelength buckling mode into the local short-wavelength buckling, thus enhancing the structural performance of the spindle. But on the other hand, the shortwavelength buckling mode is much more sensitive to the irregularities in the bundle geometry resulting in the effect of localisation of high relative displacement amplitudes towards either of the poles which presumably may affect the capacity of the cell to undergo symmetric division. Nevertheless, a cell still possesses a very reliable division mechanism which obviously should have some means of correcting the left-right symmetry breaking. This mechanism is not yet fully understood but we believe that the whole cell mechanical model may enrich our understanding of mitosis. We aim to create one such model in the future by extending the current application.

Interestingly, for $k=200 \mathrm{~Pa}$, we have observed another type of mode which has not been reported before. For some bundle morphologies when the support stiffness is high it is energetically favourable not only to shorten the buckling wavelength but also to develop another type of deformation such as torsion. Such behaviour represents a bending- torsion deformation coupling and can be observed only in three-dimensional modes. The torsional part of the buckling mode arises from the rotation of the inter-polar bundle cross-section about the axis that goes through the shear centre of the cross-section. The actual three-dimensional view of such a mode along with its projections on $x z$ and $y z$ planes discarding the aMTs are shown in Figure 4 (A). The view on the inter-polar bundle from the $x y$ plane is shown in Figure 4 (B). The direction of cross-section rotation is indicated by the grey arrow. Notice, that the inter-polar MTs deform in the helix-like fashion having both bending and torsion components to the deformation.

It should be also emphasised that due to the random positioning of

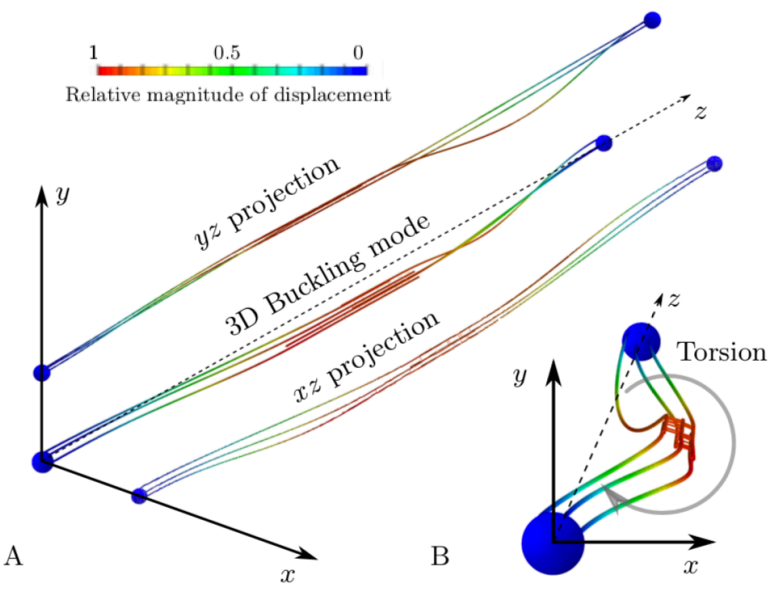

Figure 4. Three-dimensional buckling mode is depicted with its $x z$ and $y z$ projections in (A). The $x y$ view of the buckling mode showing the inter-polar MT bundle cross-section rotation and the development of helical buckling shape due to torsion is shown in (B).

MTs and cross-linkers mitotic spindles vary considerably in stiffness and structural morphology which, in turn, affect their critical loads and buckling mode shapes. The reported results are preliminary as many more computations are needed to gather reliable statistics. Yet, the application testing and comparison of the modelling results with ones reported by Ward et al. [2] indicates the potential for our proposed method to address the complex questions of spindle structural performance and of cell mitosis as a whole.

\section{CONCLUSIONS}

In this paper, we have discussed a generic modular application focused on studying the structural stability of a mitotic spindle in cell division based on the continuum mechanics approach using 
finite element analysis. The application is created in Python 2.7 programming language and employs commercial FE code Abaqus CAE via Abaqus python API. The application can be easily applied to study various mitotic spindles provided that relevant experimental data is given. Large-scale parametric studies can be easily performed as the application uses a highly optimised FE solver that can be easily multithreaded. The application is easy to use as all the essential parameters need to be provided in a single file "job.py" and it can be also easily extended due to the modulebased architecture. We have tested the application on the anaphase $\mathrm{B}$ spindle stability of the fission yeast cells and compared the buckling modes with ones reported in [2].

We have applied Spindle FEA to studying the effect of lateral reinforcement of the inter-polar MT bundle on the left-right symmetry of a mitotic spindle buckling. We have also reported a new type of buckling mode of the inter-polar MT bundle that can be observed only in $3 \mathrm{D}$ analysis and employs bending-torsion coupled deformation mechanism. Further testing and improving the application to facilitate user experience as well as to extend a range of application applicability is planned for the future work.

\section{ACKNOWLEDGEMENTS}

We would like to acknowledge Svitlana Braichenko and Lasse Wollatz for helpful discussions on developing the Spindle FEA application.

\section{REFERENCES}

[1] Dogterom, M. and Surrey, T. 2013. Microtubule organization in vitro. Current Opinion in Cell Biology, 25, 23-9. DOI=https://doi.org/10.1016/j.ceb.2012.12.002

[2] Ward, J.J., Roque, H., Antony, C. and Nédélec, F. 2014. Mechanical design principles of a mitotic spindle. ELife, 3, e03398. DOI=https://doi.org/10.7554/eLife.03398

[3] Nixon, F.M., Gutiérrez-Caballero, C., Hood, F.E., Booth, D.G., Prior, I.A. and Royle, S.J. 2015. The mesh is a network of microtubule connectors that stabilizes individual kinetochore fibers of the mitotic spindle. ELife, 4, 1-21. DOI=https://doi.org/10.7554/eLife.07635

[4] Chaigne, A., Campillo, C., Voituriez, R., Gov, N.S., Sykes, C., Verlhac, M.-H. et al. 2016. F-actin mechanics control spindle centring in the mouse zygote. Nature Communications, 7, 10253. DOI=https://doi.org/10.1038/ncomms 10253

[5] Kasas, S., Kis, A., Riederer, B.M., Forró, L., Dietler, G. and Catsicas, S. 2004. Mechanical Properties of Microtubules Explored Using the Finite Elements Method.

ChemPhysChem, 5, 252-7.

DOI=https://doi.org/10.1002/cphc. 200300799

[6] Jin, M.Z. and Ru, C.Q. 2013. Localized buckling of a microtubule surrounded by randomly distributed cross linkers. Physical Review E - Statistical, Nonlinear, and Soft Matter Physics, 88.

DOI=https://doi.org/10.1103/PhysRevE.88.012701

[7] Rubinstein, B., Larripa, K., Sommi, P. and Mogilner, A. 2009. The elasticity of motor-microtubule bundles and shape of the mitotic spindle. Physical Biology, 6, 016005. DOI=https://doi.org/10.1088/1478-3975/6/1/016005

[8] Malgaretti, P. and Muhuri, S. 2016. Mechanical stability of bipolar spindle assembly. EPL (Europhysics Letters), 115, 28001. DOI=https://doi.org/10.1209/0295-5075/115/28001

[9] Kellogg, E.H., Howes, S., Ti, S.-C., Ramírez-Aportela, E., Kapoor, T.M., Chacón, P. et al. 2016. Near-atomic cryoEM structure of PRC1 bound to the microtubule.
Proceedings of the National Academy of Sciences, 113, 9430-9439. DOI=https://doi.org/10.1073/pnas.1609903113

[10] Pampaloni, F., Lattanzi, G., Jonas, A., Surrey, T., Frey, E. and Florin, E.-L. 2006. Thermal fluctuations of grafted microtubules provide evidence of a length-dependent persistence length. Proceedings of the National Academy of Sciences, 103, 10248-10253. DOI=https://doi.org/10.1073/pnas.0603931103

[11] Wang, C.Y., Ru, C.Q. and Mioduchowski, A. 2006. Orthotropic elastic shell model for buckling of microtubules. Physical Review E, 74, 052901. DOI=https://doi.org/10.1103/PhysRevE.74.052901

[12] Van den Heuvel, M.G.L., De Graaff, M.P. and Dekker, C. 2008. Microtubule curvatures under perpendicular electric forces reveal a low persistence length. Proceedings of the National Academy of Sciences, 105, 7941-7946. DOI=https://doi.org/10.1073/pnas.0704169105

[13] Liew, K.M., Xiang, P. and Sun, Y. 2011. A continuum mechanics framework and a constitutive model for predicting the orthotropic elastic properties of microtubules. Composite Structures, 93, 1809-18. DOI=https://doi.org/10.1016/j.compstruct.2011.01.017

[14] Soheilypour, M., Peyro, M., Peter, S.J. and Mofrad, M.R.K. 2015. Buckling behavior of individual and bundled microtubules. Biophysical Journal, 108, 1718-1726. DOI=https://doi.org/10.1016/j.bpj.2015.01.030

[15] Brangwynne, C.P., MacKintosh, F.C., Kumar, S., Geisse, N.A., Talbot, J., Mahadevan, L. et al. 2006. Microtubules can bear enhanced compressive loads in living cells because of lateral reinforcement. Journal of Cell Biology, 173, 733-741. DOI=https://doi.org/10.1083/jcb.200601060

[16] di Pietro, F., Echard, A. and Morin, X. 2016. Regulation of mitotic spindle orientation: an integrated view. $E M B O$ Reports, 17, 1106-30. DOI=https://doi.org/10.15252/embr.201642292

[17] Müller-Reichert, T., Kiewisz, R. and Redemann, S. 2018. Mitotic spindles revisited - new insights from 3D electron microscopy. Journal of Cell Science, 131, jcs211383. DOI=https://doi.org/10.1242/jcs. 211383

[18] Pavin, N. and Tolić, I.M. 2016. Self-Organization and Forces in the Mitotic Spindle. Annual Review of Biophysics, 45, 279-98. DOI=https://doi.org/10.1146/annurev-biophys062215-010934

[19] Nedelec, F. and Foethke, D. 2007. Collective Langevin dynamics of flexible cytoskeletal fibers. New Journal of Physics, 9. DOI=https://doi.org/10.1088/13672630/9/11/427

[20] Jakobs, M., Franze, K. and Zemel, A. 2015. Force Generation by Molecular-Motor-Powered Microtubule Bundles; Implications for Neuronal Polarization and Growth. Frontiers in Cellular Neuroscience, 9, 4413389441. DOI=https://doi.org/10.3389/fncel.2015.00441

[26] A. Iakovliev, Spindle FEA. University of Southampton. DOI=https://doi.org/10.5258/SOTON/D0603

[22] Kabir, A.M.R., Inoue, D., Afrin, T., Mayama, H., Sada, K. and Kakugo, A. 2015. Buckling of Microtubules on a 2D Elastic Medium. Scientific Reports, 5, 17222. DOI=https://doi.org/10.1038/srep 17222

[23] Timoshenko, S.P.S. and Gere, J.M.J. 2012. Theory of elastic stability [Internet]. Courier Corporation.

[24] Gittes, F., Mickey, B., Nettleton, J. and Howard, J. 1993. Flexural rigidity of microtubules and actin filaments measured from thermal fluctuations in shape. The Journal 
of Cell Biology, 120, 923-934. DOI=https://doi.org/DOI: 10.1083/jcb.120.4.923

[25] Janson, M.E. and Dogterom, M. 2004. A bending mode analysis for growing microtubules: evidence for a velocitydependent rigidity. Biophysical Journal, 87, 2723-2736. DOI=https://doi.org/10.1529/biophysj.103.038877

[26] Claessens, M., Bathe, M., Frey, E. and Bausch, A.R. 2006. Actin-binding proteins sensitively mediate F-actin bundle stiffness. Nature Materials, 5, 748-753.

DOI=https://doi.org/10.1038/nmat 1718

[27] Subramanian, R., Wilson-Kubalek, E.M., Arthur, C.P., Bick, M.J., Campbell, E.A., Darst, S.A. et al. 2010. Insights into Antiparallel Microtubule Crosslinking by PRC1, a Conserved Nonmotor Microtubule Binding Protein. Cell, 142, 433-43.

DOI=https://doi.org/10.1016/j.cell.2010.07.012

\section{APPENDIX}

\section{A. GEOMETRIC AND PHYSICAL} PARAMETERS OF THE SPINDLE

The geometric and physical parameters of the spindle employed in the study and supplied to "job.py" file located within the root directory of the application are presented in Table A. The first column contains the explanation of the parameter, the second column contains name of the variable representing the parameter and the third column contains parameter values. The content of the "job.py" as well as the rules to modify it can be found in the interactive documentation to the Spindle FEA [21].

Table A. Mitotic spindle parameters

\begin{tabular}{|c|c|c|}
\hline Name & Name in "job.py" & Values \\
\hline \multicolumn{3}{|c|}{ Spindle architecture } \\
\hline $\begin{array}{l}\text { Total spindle length } \\
\text { [2] }\end{array}$ & - & $14.3 \mu \mathrm{m}$ \\
\hline Cell radius [2] & - & $1.6 \pm 1 \mu \mathrm{m}$ \\
\hline $\begin{array}{l}\text { Inter-pole distance } \\
{[2]}\end{array}$ & SpindleLength & $10 \mu \mathrm{m}$ \\
\hline $\begin{array}{l}\text { Length of } \\
\text { interconnected zone } \\
{[2]}\end{array}$ & - & $2 \mu \mathrm{m}$ \\
\hline \multicolumn{3}{|c|}{ Microtubules } \\
\hline $\begin{array}{l}\text { MT inner diameter } \\
{[10]}\end{array}$ & $d$ & $0.015 \mu \mathrm{m}$ \\
\hline $\begin{array}{l}\text { MT outer diameter } \\
{[10]}\end{array}$ & D & $0.025 \mu \mathrm{m}$ \\
\hline $\begin{array}{l}\text { Inter-polar MT } \\
\text { length defined by } \\
\text { Gaussian } \\
\text { distribution }\end{array}$ & lengthInterval & $\begin{aligned} \sigma & =5 \mu \mathrm{m}, \\
\mu & =2 \mu \mathrm{m}\end{aligned}$ \\
\hline
\end{tabular}

\begin{tabular}{|c|c|c|}
\hline Astral MT length & aMTlength & $2 \mu \mathrm{m}$ \\
\hline $\begin{array}{l}\text { Number of ipMTs } \\
\text { [2] }\end{array}$ & ipMTnumber & 6 \\
\hline Number of aMTs & aMTnumbers & 20 \\
\hline $\begin{array}{l}\text { Distance between } \\
\text { ipMTs, distribution } \\
\text { fitted to [2] }\end{array}$ & separation & $\begin{aligned} \sigma & =0.029 \mu \mathrm{m}, \\
\mu & =0.04 \mu \mathrm{m}\end{aligned}$ \\
\hline $\begin{array}{l}\text { Angle between } \\
\text { ipMTs, distribution } \\
\text { fitted to [2] }\end{array}$ & angle & $\begin{array}{l}\sigma=96.4^{\circ} \\
\mu=11.1^{\circ}\end{array}$ \\
\hline $\begin{array}{l}\text { Elastic modulus of } \\
\text { MTs [10] }\end{array}$ & ElasticModulus & $1.5 \times 10^{9} \frac{\mathrm{pN}}{\mu \mathrm{m}^{2}}$ \\
\hline $\begin{array}{l}\text { Assumed Poisson } \\
\text { ratio of MT material } \\
\text { for isotropic case }\end{array}$ & PoissonRatio & 0.3 \\
\hline \multicolumn{3}{|c|}{ Centrosomes } \\
\hline Centrosome radius & $\begin{array}{c}\text { CentrosomeRadiu } \\
\mathbf{s}\end{array}$ & $240 \mathrm{~nm}$ \\
\hline Centrosome length & $\begin{array}{c}\text { CentrosomeLengt } \\
\mathrm{h}\end{array}$ & $480 \mathrm{~nm}$ \\
\hline $\begin{array}{l}\text { Elastic modulus of } \\
\text { centrosome material }\end{array}$ & CentrosomeE & $1.5 \times 10^{9} \frac{\mathrm{pN}}{\mu \mathrm{m}^{2}}$ \\
\hline $\begin{array}{l}\text { Poisson ratio } \\
\text { of centrosome } \\
\text { material }\end{array}$ & Centrosomenu & 0.3 \\
\hline \multicolumn{3}{|c|}{ Connectors } \\
\hline Connector radius & connectorRadius & $5 \mathrm{~nm}$ \\
\hline $\begin{array}{l}\text { Connector length } \\
{[27]}\end{array}$ & - & $20-50 \mathrm{~nm}$ \\
\hline $\begin{array}{l}\text { Number of } \\
\text { connectors per MT }\end{array}$ & Nconnectors & 10 \\
\hline $\begin{array}{l}\text { Elastic modulus of } \\
\text { connector material }\end{array}$ & connectore & $1.5 \times 10^{9} \frac{\mathrm{pN}}{\mu \mathrm{m}^{2}}$ \\
\hline $\begin{array}{l}\text { Poisson ratio } \\
\text { of connector } \\
\text { material }\end{array}$ & connectorNu & 0.3 \\
\hline \multicolumn{3}{|c|}{ Other parameters } \\
\hline $\begin{array}{lr}\text { Astral } & \text { MT } \\
\text { connecting } & \text { spring } \\
\text { stiffness } & \\
\end{array}$ & aMTspring & $0-200 \frac{\mathrm{pN}}{\mu \mathrm{m}^{2}}$ \\
\hline $\begin{array}{l}\text { Inter-polar bundle } \\
\text { supporting spring }\end{array}$ & groundSpring & $0-200 \frac{\mathrm{pN}}{\mu \mathrm{m}^{2}}$ \\
\hline $\begin{array}{ll}\text { Number } & \text { of } \\
\text { requested } & \\
\text { eigenvalues } & \end{array}$ & NumberOfEigs & 5 \\
\hline
\end{tabular}

\section{Columns on Last Page Should Be Made As Close As Possible to Equal Length}

